\title{
Seed Populations for Large Solar Particle Events Of Cycle 23
}

\author{
M. I. Desai ${ }^{\mathrm{a}}$, G. M. Mason ${ }^{\mathrm{b}}$, R. E. Gold ${ }^{\mathrm{b}}$, S. M. Krimigis ${ }^{\mathrm{b}}$, C. M. S. \\ Cohen $^{\mathrm{c}}$, R. A. Mewaldt ${ }^{\mathrm{c}}$, J. R. Dwyer ${ }^{\mathrm{d}}$, and J. E. Mazur ${ }^{\mathrm{e}}$ \\ ${ }^{a}$ Southwest Research Institute, 6220 Culebra Road, San Antonio, TX 78238 \\ ${ }^{b}$ Johns Hopkins University/Applied Physics Laboratory, Laurel, MD 20723 \\ ${ }^{\circ}$ California Institute of Technology, Pasadena, CA 91125 \\ ${ }^{d}$ Florida Institute of Technology, Melbourne, FL 32901 \\ The Aerospace Corporation, Chantilly, VA 20151
}

\begin{abstract}
Using high-resolution mass spectrometers on board the Advanced Composition Explorer (ACE), we surveyed the event-averaged $\sim 0.1-60 \mathrm{MeV} /$ nuc heavy ion elemental composition in 64 large solar energetic particle (LSEP) events of cycle 23. Our results show the following: (1) The rare isotope ${ }^{3} \mathrm{He}$ is greatly enhanced over the corona or the solar wind values in $46 \%$ of the events. (2) The $\mathrm{Fe} / \mathrm{O}$ ratio decreases with increasing energy up to $\sim 10 \mathrm{MeV} / \mathrm{nuc}$ in $\sim 92 \%$ of the events and up to $\sim 60 \mathrm{MeV} / \mathrm{nuc}$ in $\sim 64 \%$ of the events. (3) Heavy ion abundances from $\mathrm{C}-\mathrm{Fe}$ exhibit systematic $M / Q$-dependent enhancements that are remarkably similar to those seen in ${ }^{3}$ He-rich SEP events and CME-driven interplanetary (IP) shock events. Taken together, these results confirm the role of shocks in energizing particles up to $-60 \mathrm{MeV} / \mathrm{nuc}$ in the majority of large SEP events of cycle 23 , but also show that the seed population is not dominated by ions originating from the ambient corona or the thermal solar wind, as previously believed. Rather, it appears that the source material for CME-associated large SEP events originates predominantly from a suprathermal population with a heavy ion enrichment pattern that is organized according to the ion's mass-per-charge ratio. These new results indicate that current LSEP models must include the routine production of this dynamic suprathermal seed population as a critical pre-cursor to the CME shock acceleration process.
\end{abstract}

Keywords: Solar Energetic Particles, -- Particle Acceleration - Sun -- Solar Cycle

PACS: 96.50.-e; 96.50.Pw; 96.50.sb

\section{INTRODUCTION}

Through much of the 1970's - 1990's, the near-Earth observations of energetic particle events from the Sun (SEPs) were grouped into two classes - impulsive and gradual. In this two-class picture the gradual events occurred as a result of diffusive acceleration of ambient coronal or solar wind material at CME-driven coronal and interplanetary (IP) shocks, while the impulsive events were attributed to stochastic acceleration of coronal material heated up to $\sim 10 \mathrm{MK}$ during magnetic reconnection in solar flares (see review [1]). The gradual or CME shock-accelerated events lasted several days and had larger fluences, while the impulsive or flare-accelerated events lasted a few hours and had smaller fluences. Impulsive events were observed when the observer was magnetically connected to the flare site, while ions associated with large-

\footnotetext{
CP1039, Particle Acceleration and Transport in the Heliosphere and Beyond $7^{\text {th }}$ Annual Astrophysics Conference edited by G. Li, Q. Hu, O. Verkhoglyadova, G. P. Zank, R. P. Lin, and J. Luhmann (O) 2008 American Institute of Physics 978-0-7354-0566-0/08/\$23.00
} 
scale CME-driven shocks populate magnetic field lines over a broad range of longitudes [2]. The impulsive events were electron-rich and associated with Type III radio bursts. These events also had ${ }^{3} \mathrm{He} /{ }^{4} \mathrm{He}$ ratios enhanced between factors of $10^{3}-$ $10^{4}$ and $\mathrm{Fe} / \mathrm{O}$ ratios enhanced by up to a factor of 10 over the corresponding solar wind values, and had $\mathrm{Fe}$ with ionization states up to $\sim 20$. In contrast, the gradual events were proton-rich, were associated with Type II bursts, had average $\mathrm{Fe} / \mathrm{O}$ ratios of $\sim 0.1$ with $\mathrm{Fe}$ ionization states of $\sim 14$, and were assumed to have ${ }^{3} \mathrm{He} /{ }^{4} \mathrm{He}$ ratios similar to the solar wind value $[1,3]$.

Since these earlier studies, instruments with greater sensitivity and resolution on board the Advanced Composition Explorer (ACE) [4] have provided major observational advances in terms of measuring (1) the solar wind ion composition and its variations [5-6] and (2) the energy-dependence and event-to-event variability of the ionic charge states, elemental, and isotopic composition in SEP events over a broad energy range [7-12]. These new observations have made it possible to re-examine the origin of the seed populations and probe details of the acceleration and transport mechanisms for individual large SEP events. In this paper, we discuss key observations that have spawned a re-evaluation of a key aspect of large SEP events that of the origin of the seed populations.

\section{OBSERVATIONS}

We use data from the Ultra-Low-Energy Isotope Spectrometer (ULEIS, [13]) and the Solar Isotope Spectrometer (SIS, [14]) on board ACE. We selected 64 large SEP (LSEP) events from NOAA's list of 85 solar proton events (SPEs) that affected Earth's environment between November 1997 and January 2005. For details of the event selection and their sampling intervals see [10].

\section{${ }^{3}$ He Enhancements}

Figure la shows the $0.5-2.0 \mathrm{MeV} / \mathrm{nuc}^{3} \mathrm{He}$ and ${ }^{4} \mathrm{He}$ time-intensity profiles in a large SEP event that occurred on June 4, 1999 (from [15]). The temporal profiles of the two species are remarkably similar, which indicates that they probably have the same acceleration and transport history. In this event, the ${ }^{3} \mathrm{He}$ is enriched by a factor of $16 \pm$ 3 while the $\mathrm{Fe} / \mathrm{O}$ ratio (not shown) is enhanced by about a factor of 10 over the corresponding solar wind values.

In this survey, we find that the $0.5-2.0 \mathrm{MeV} / \mathrm{nuc}{ }^{3} \mathrm{He} /{ }^{4} \mathrm{He}$ ratio in 29 of the 64 events $(\sim 46 \%)$ is enhanced between factors $\sim 4-150$ over the corresponding solar wind value. Large enrichments in the ${ }^{3} \mathrm{He} /{ }^{4} \mathrm{He}$ ratio are also observed above $\sim 10 \mathrm{MeV} / \mathrm{nuc}$ [16-17]. Figure 1b shows the $\sim 1 \mathrm{MeV} / \mathrm{nuc} \mathrm{He}$ mass histogram from several such events. Notice that the ${ }^{3} \mathrm{He}$ is clearly resolved from ${ }^{4} \mathrm{He}$ and the background. 

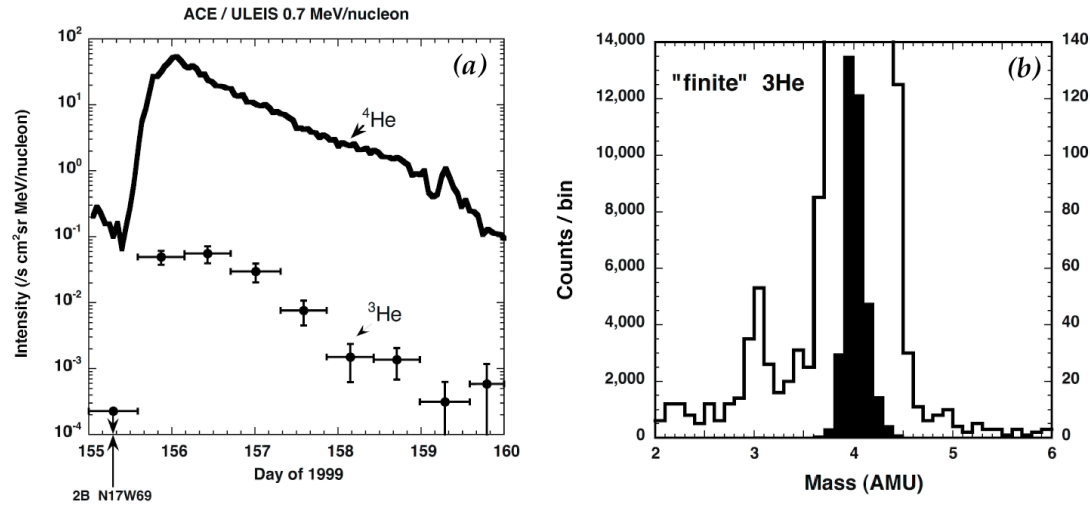

FIGURE 1: (a) Temporal profiles of $\sim 0.7 \mathrm{MeV} / \mathrm{nuc}^{3} \mathrm{He}$ and ${ }^{4} \mathrm{He}$ ions in a large SEP event. (b) $0.5-2.0$ $\mathrm{MeV} / \mathrm{nuc}$ He mass histogram obtained during several large SEP events. The right scale corresponds to the open histogram (taken from [15]).

\section{Energy-Dependence of $\mathbf{F e} / \mathrm{O}$}

We compared (see [12]) the average LSEP abundances with the average slow solar wind values as a function of M/Q or First Ionization Potential (FIP), and found that the LSEP abundances at $\sim 0.4 \mathrm{MeV} /$ nuc were poorly correlated with the solar wind values. In particular, we found that elements with similar mass-per-charge $(M / Q)$ values like $\mathrm{C}, \mathrm{N}, \mathrm{O}, \mathrm{Ne}$, and $\mathrm{Mg}$ exhibited highly unsystematic enhancements and depletions relative to the corresponding solar wind values, with the $\mathrm{C} / \mathrm{O}$ ratio in particular being depleted by about a factor of 2 .

Figure 2 shows the energy-dependent behavior of $\mathrm{Fe} / \mathrm{O}$ in 37 of the 64 large SEP events in this survey. The $\mathrm{Fe} / \mathrm{O}$ ratios are $0.11-0.14 \mathrm{MeV} / \mathrm{nuc}$ from ULEIS [12]; $3.3-$ $10 \mathrm{MeV} / \mathrm{nuc}$ from LEMT [18]; and 12-60 MeV/nuc from SIS [12]. Note that the Fe/O ratio either decreases or remains constant with increasing energy in 34 of the 37 events $(-92 \%)$ up to $\sim 10 \mathrm{MeV} / \mathrm{nuc}$ and in 26 of the 36 events $(\sim 64 \%)$ up to $\sim 60 \mathrm{MeV} / \mathrm{nuc}$. However, an unexpected and puzzling feature of Fig. $2 \mathrm{a}$ is that the low-energy $\mathrm{Fe} / \mathrm{O}$ ratio between $0.11-0.14 \mathrm{MeV} /$ nuc in most of the LSEP events (55 of the 64 events; see [12]) is enhanced when compared with the average solar wind value.

\section{Mass-per-Charge Dependent Enhancements}

In order to examine whether the large enhancements and the event-to-event variability seen in the low-energy $\mathrm{Fe} / \mathrm{O}$ are systematically organized by the ion's $M / Q$ ratio, we plot in Fig. 3: (a) the $\mathrm{S} / \mathrm{O}$ ratio and (b) the $\mathrm{Ca} / \mathrm{O}$ ratio versus the $\mathrm{Fe} / \mathrm{C}$ ratio at $\sim 0.38 \mathrm{MeV} /$ nuc measured in the $64 \mathrm{LSEP}$ events in the survey. We chose to plot the event-averaged abundances of S, Ca, and Fe (i.e., low FIP elements) relative to $\mathrm{C}$ and $\mathrm{O}$ (i.e., high FIP elements) to minimize the so-called FIP fractionation effect seen in large SEP events [19].

Figure 3 also compares the LSEP abundances with those seen in IP shock events of [20] and ${ }^{3} \mathrm{He}$-rich SEP events of [21]. The dashed line represents a linear fit to the LSEP data with slope $m$ and intercept $c$. We remark that the slopes and intercepts of 

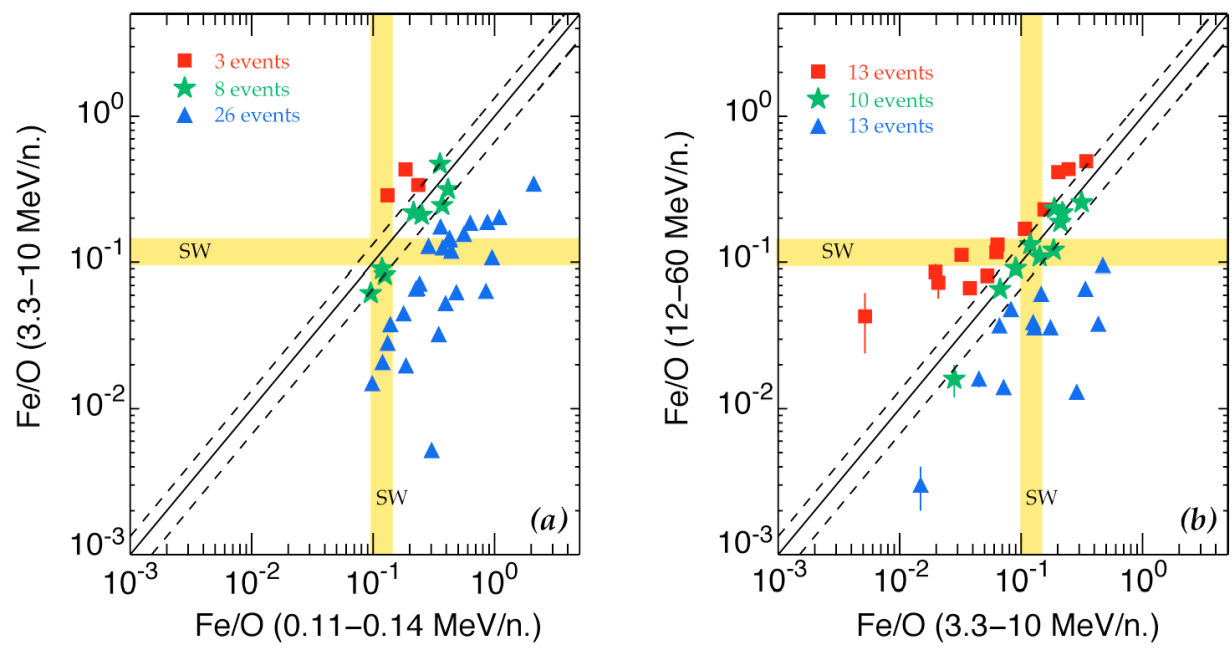

FIGURE 2: (a) $\mathrm{Fe} / \mathrm{O}$ ratio measured at $0.11-0.14 \mathrm{MeV} /$ nuc versus that measured at $3.3-10 \mathrm{MeV} / \mathrm{nuc}$ for 37 large SEP events. (b) $\mathrm{Fe} / \mathrm{O}$ ratio measured at 3.3-10 MeV/nuc versus that measured at 12-60 $\mathrm{MeV} / \mathrm{nuc}$ for $36 \mathrm{SEP}$ events. Yellow bands represent error limits for the $\mathrm{Fe} / \mathrm{O}$ ratio measured in the slow solar wind (taken from [12]).

the linear fits obtained independently by fitting the data for IP shocks (blue) and ${ }^{3} \mathrm{He}-$ rich events (green) are well within the respective $1 \sigma$ error limits of the fit parameters for the three types of events.

Figure 3 shows the following: (1) Enhancements in $\mathrm{S} / \mathrm{O}$ and $\mathrm{Ca} / \mathrm{O}$ are accompanied by simultaneous enhancements in the $\mathrm{Fe} / \mathrm{C}$ ratio. (2) The fits to the data provide an excellent representation of the event-to-event variations and the enhancement pattern of the heavy ion abundances in large SEP events. (3) For each element, the power-law dependence in large SEP events provides remarkably good fits to the corresponding event-to-event variations seen in IP shock events and in ${ }^{3} \mathrm{He}$-rich SEP events.

\section{DISCUSSION}

Our survey of the $\sim 0.1-60 \mathrm{MeV} /$ nuc heavy ion abundances in 64 LSEP events of cycle 23 shows the following:

1. ${ }^{3} \mathrm{He}$ is enhanced over the corona or the solar wind values in $46 \%$ of the events.

2. The $\mathrm{Fe} / \mathrm{O}$ ratio decreases with increasing energy in $\sim 92 \%$ of the events up to $\sim 10$ $\mathrm{MeV} / \mathrm{nuc}$ and in $\sim 64 \%$ of the events up to $\sim 60 \mathrm{MeV} / \mathrm{nuc}$.

3. Event-to-event variations in the heavy ion abundances in LSEP events are organized according to the $M / Q$ ratio and are remarkably similar to those seen in ${ }^{3} \mathrm{He}$-rich SEP events and IP shock events. 

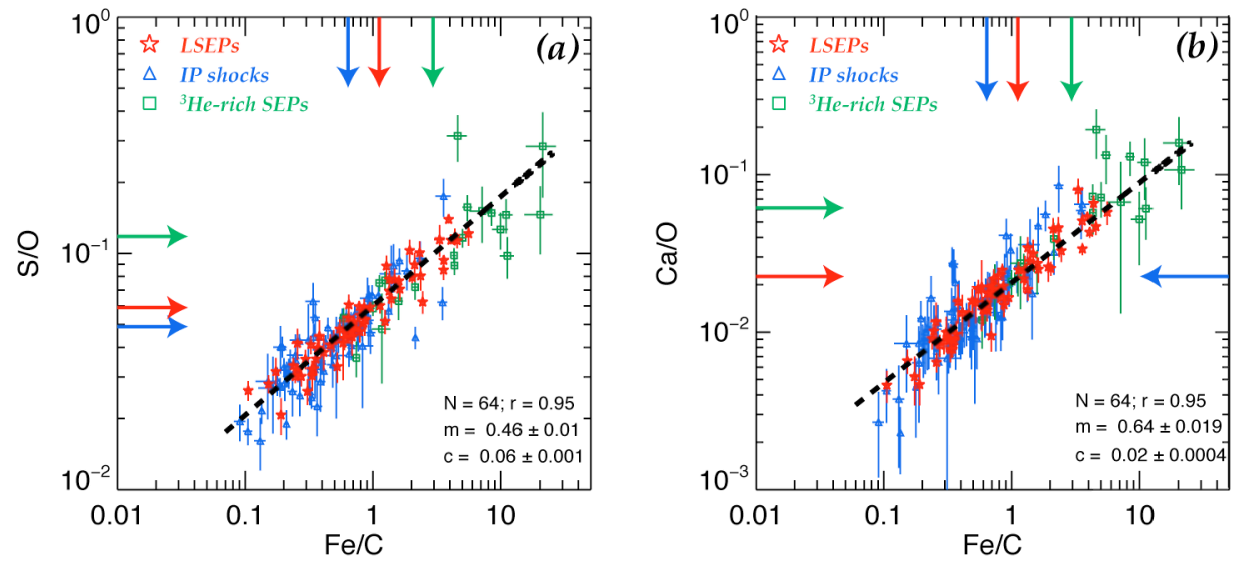

FIGURE 3: (a) $\mathrm{S} / \mathrm{O}$ and (b) $\mathrm{Ca} / \mathrm{O}$ plotted versus $\mathrm{Fe} / \mathrm{C}$ ratio at $0.38 \mathrm{MeV} / \mathrm{nuc}$ for three different types of events. Blue triangles = IP shock events of [20]; red asterisks = large SEP events in this survey; and green squares $={ }^{3} \mathrm{He}$-rich SEP events of [21]. Colored arrows along the axes represent the average values of the respective populations. Dashed black line represents the linear fit to the large SEP data. The quantities $N$ and $r$ represent the number of SEP events and the correlation coefficient. The quantities $m$ and $c$ represent the slopes and intercepts of the linear fits (taken from [10]).

\section{Two-stage Acceleration in Large SEP Events}

Rigidity-dependent shock acceleration processes tend to deplete the abundances of heavier ions when compared with the lighter ions (e.g., [20, 22-24]). Fig. 2 shows that the $\mathrm{Fe} / \mathrm{O}$ ratio decreases with increasing energy in the majority of the LSEP events in our survey. Such systematic energy dependence of the SEP Fe/O ratios were also reported by a number of previous studies [9, 25-27]. Likewise, the $\mathrm{Fe} / \mathrm{O}$ ratios in IP shock events at ACE also exhibited similar properties [20, 24], and are consistent with expectations from shock acceleration models [28-29] where ions with higher $M / Q$ ratios are accelerated less efficiently than those with lower $M / Q$ ratios.

However, the ${ }^{3} \mathrm{He} /{ }^{4} \mathrm{He}$ ratio (Fig. 1b) and the heavy ion abundances in large SEP events (Fig. 2a) are significantly enhanced relative to the slow solar wind or the ambient coronal abundances $[10,15-16]$. Since ${ }^{3} \mathrm{He}$ has a smaller $M / Q$ ratio than ${ }^{4} \mathrm{He}$ while ions such as $\mathrm{Fe}$ have a larger $M / Q$ ratio than $\mathrm{O}$, such large simultaneous enrichments are difficult to reconcile with rigidity-dependent shock acceleration of solar wind material. This implies that the dominant seed population for the majority of the large SEP events could not have originated either from the thermal or suprathermal solar wind or from the ambient corona [27, 30]. In fact, Figs. 2 and 3 point to the occurrence of two distinct $M / Q$ dependent fractionation processes in the same large SEP event. The first process results in producing $M / Q$-dependent enhancements, similar to those seen in the ${ }^{3} \mathrm{He}$-rich SEP events, while the second process-probably due to shock acceleration-causes the $\mathrm{Fe} / \mathrm{O}$ ratio to decrease with increasing energy.

Figure $2 \mathrm{~b}$ also shows that for $\sim 36 \%$ of the events there is a decrease in $\mathrm{Fe} / \mathrm{O}$ around $\sim 3-10 \mathrm{MeV} / \mathrm{nuc}$ followed by an increase at the higher energies. This behavior is puzzling and could be due to a separate mechanism operating at these high energies e.g., a direct Fe-rich acceleration event near the Sun [31, 32], or perhaps the re- 
acceleration of a flare seed population with $\mathrm{Fe} / \mathrm{O}$ ratios that increase with energy [26]. No doubt other possibilities exist, and we believe that there is insufficient data to resolve the issue at this time.

Using the fact that the heavy ion abundances in CME-driven IP shocks at $1 \mathrm{AU}$ [20] are depleted systematically according to the ion's $M / Q$ ratio when compared with the ambient suprathermal population measured prior to the arrival of the shocks, [10] suggested that the average abundances for the "source" population for LSEPs may be even more abundant in heavier ions than the observed abundances in LSEPs and could resemble that seen in impulsive SEPs. These new results indicate that current LSEP models must include the routine production of a dynamic suprathermal or preaccelerated seed population as a critical pre-cursor to CME shock acceleration.

\section{ACKNOWLEDGEMENTS}

Work at SwRI is partially supported by NSF grants ATM-0550960 and ATM0551127 and NASA grants: NNG05GM88G, NNG05GQ94G, NNX07AC12G, NNX07AG85G, NNX07AP69G, and NNX08AK87G. We thank the following organizations for permission to publish copyrighted material: Elsevier (Fig. 2, from [12]); and American Astronomical Society (Fig. 1 from [15] and Fig. 3 from 10]).

\section{REFERENCES}

1. D.V. Reames, Space Sci. Rev. 90, 413 (1999)

2. H.V. Cane, D.V. Reames, T.T. von Rosenvinge, Astrophys. J. 93, 9555 (1988)

3. E.W. Cliver, AIP Conference Proceedings, vol. 528, 21 (2000)

4. E.C. Stone, et al., Space Sci. Rev. 86, 1 (1998a)

5. G. Gloeckler et al., Astron. Astrophys. Suppl. Ser. 92, 267 (1992)

6. J. Geiss, G. Gloeckler, R. von Steiger, Space Sci. Rev. 78, 43 (1996)

7. J.E. Mazur et al., Geophys. Res. Lett. 26, 173 (1999)

8. E. Möbius et al., Geophys. Res. Lett. 26, 145 (1999)

9. C.M.S. Cohen et al., J. Geophys. Res. 110, A09S16 (2005)

10. M.I. Desai et al., Astrophys. J. 649, 470 (2006)

11. B. Klecker et al., Space Sci. Rev. 124(1-4), 289 (2006)

12. M.I. Desai et al., Space Sci. Rev. 649, 470 (2007)

13. G.M. Mason et al., Space Sci. Rev. 86, 409 (1998)

14. E.C. Stone et al., Space Sci. Rev. 86, 285-356 (1998b)

15. G.M. Mason, J.E. Mazur, J.R. Dwyer, Astrophys. J. Lett. 525, L133 (1999)

16. C.M.S. Cohen et al., Geophys. Res. Lett. 26, 2697 (1999)

17. M. E. Wiedenbeck et al., AIP, CP, 528, 107, (2000)

18. D.V. Reames, C.K. Ng, Astrophys. J. 610, 510 (2004)

19. R.A. Mewaldt et al., Space Sci. Rev., doi:10.1007/s11214-007-9187-1 (2007)

20. M.I. Desai et al., Astrophys. J. 558, 1149 (2003)

21. G.M. Mason et al., Astrophys. J. 606, 555 (2004)

22. B. Klecker et al., Astrophys. J. 251, 391 (1981)

23. H.V. Cane, D.V. Reames, T.T. von Rosenvinge, Astrophys. J. 373, 675 (1991)

24. M.I. Desai et al., Astrophys. J. 611, 1156 (2004)

25. J.E. Mazur et al., Astrophys. J. 401, 398 (1992)

26. A. J. Tylka et al., Astrophys. J. 625, 474 (2005)

27. R.A. Mewaldt, C.M.S. Cohen, G.M. Mason, in Geophys. Monograph Series, vol. 165, 115, (2006)

28. M.A. Lee, Astrophys. J. 158, 38 (2005) 
29. G. Li, et al., J. Geophys. Res., 110, A06104 (2005)

30. R.A. Mewaldt et al., Adv. Space Res. 30, 79 (2002)

31. H. V. Cane, et al., Geophys. Res. Lett. 30, Issue 12, SEP 5-1, (2003)

32. H. V. Cane, et al., J. of Geophys. Res. 111, Issue A6, doi: 10.1029/2005JA011071, (2006) 